\title{
HOLOMORPHIC MORSE INEQUALITIES IN SINGULAR REDUCTION
}

\author{
YOULIANG TIAN AND WEIPING ZHANG
}

Abstract. We extend our Morse type inequalities for holomorphic symplectic reductions $[\mathrm{TZ1}, 2]$ to the case of singular reductions.

\section{Introduction and the statement of main results}

Let $(M, \omega)$ be a closed symplectic manifold. We make the assumption that there is a Hermitian line bundle $L$ over $M$ admitting a Hermitian connection $\nabla^{L}$ with the property that $\frac{\sqrt{-1}}{2 \pi}\left(\nabla^{L}\right)^{2}=\omega$. When such a line bundle exists, it is usually called a prequantum line bundle over $M$. Let $J$ be an almost complex structure on $T M$ such that $g^{T M}(u, v)=\omega(u, J v)$ defines a Riemannian metric on $T M$. Then one can construct canonically a $\operatorname{Spin}^{c}$-Dirac operator

$$
D^{L}: \Omega^{0, *}(M, L) \rightarrow \Omega^{0, *}(M, L),
$$

which gives the finite dimensional virtual vector space

$$
Q(M, L)=\left(\operatorname{ker} D^{L}\right) \cap \Omega^{0, \text { even }}(M, L)-\left(\operatorname{ker} D^{L}\right) \cap \Omega^{0, \text { odd }}(M, L) .
$$

Next, suppose that $(M, \omega)$ admits a Hamiltonian action of a compact connected Lie group $G$ with Lie algebra $g$. Let $\mu: M \rightarrow g^{*}$ be the corresponding moment map. Then a formula due to Kostant [Ko] (cf. [TZ2, (1.13)]) induces a natural $g$ action on $L$. We make the assumption that this $g$ action can be lifted to a $G$ action on $L$. Then this $G$ action preserves $\nabla^{L}$. One can also assume, after an integration over $G$ if necessary, that $G$ preserves the Hermitian metric on $L$, the almost complex structure $J$ and thus also the Riemannian metric $g^{T M}$. $Q(M, L)$ then becomes a virtual representation of $G$. We denote by $Q(M, L)^{G}$ its $G$-invariant part.

Now let $a \in g^{*}$ be a regular value of $\mu$. Let $\mathcal{O}_{a} \subset g^{*}$ be the coadjoint orbit of $a$. For simplicity, we assume that $G$ acts on $\mu^{-1}\left(\mathcal{O}_{a}\right)$ freely. Then the quotient space $M_{G, a}=\mu^{-1}\left(\mathcal{O}_{a}\right) / G$ is smooth. Furthermore, $\omega$ descends canonically to a symplectic form $\omega_{G, a}$ on $M_{G, a}$ so that one gets the Marsden-Weinstein reduction $\left(M_{G, a}, \omega_{G, a}\right)$. The pair $\left(L, \nabla^{L}\right)$ also descends canonically to a Hermitian line

Received September 5, 1998.

The first author was partially supported by a NSF postdoctoral fellowship and a NYU research challenge fund grant.

The second author was partially supported by the NNSF, SEC of China and the Qiu Shi Foundation. 
bundle $L_{G, a}$ with a Hermitian connection denoted by $\nabla^{L_{G, a}} \cdot{ }^{1}$ The almost complex structure $J$ also descends canonically to an almost complex structure on $T M_{G, a}$. Thus once again one can construct canonically a Spin ${ }^{c}$-Dirac operator $D^{L_{G, a}}$ as well as the corresponding virtual vector space $Q\left(M_{G, a}, L_{G, a}\right)$.

If $0 \in g^{*}$ is a regular value of $\mu$, then the well-known geometric quantization conjecture of Guillemin and Sternberg [GS] asserts that

$$
\operatorname{dim} Q(M, L)^{G}=\operatorname{dim} Q\left(M_{G, 0}, L_{G, 0}\right) .
$$

This conjecture has been proved in various generalities in [DGMW, G, GS, JK, M1, M2, V], mainly in using the equivariant index theorem of Atiyah-SegalSinger $[\mathrm{AS}]$.

In $[\mathrm{TZ1}, 2]$, with the help of the techniques in $[\mathrm{BL}]$, we have developed a direct analytic approach to this quantization conjecture. Besides offering an alternative proof of (0.3), our analytic methods also lead immediately to further generalizations which include (i) the case where $L$ is replaced by an arbitrary $G$ equivariant Hermitian vector bundle verifying certain natural conditions and (ii) the case where $(M, \omega, J)$ is Kähler, in which one can refine the results mentioned in (i) to systems of Morse type inequalities.

On the other hand, a generalization of (0.3) to the general case where $0 \in g^{*}$ might be a singular value of $\mu$ has been given by Meinrenken-Sjamaar [MS].

The purpose of this note is to extend the Morse type inequalities mentioned above to certain cases where $0 \in \mathbf{g}^{*}$ might be a singular value of $\mu$.

For this purpose, we assume that $(M, \omega, J)$ is Kähler and that $G$ acts holomorphically on $M$. Then $L$ carries a unique holomorphic structure so that $\nabla^{L}$ is the associated Hermitian holomorphic connection. Furthermore, for each regular value $a \in \mathbf{g}^{*}$ of $\mu,\left(M_{G, a}, \omega_{G, a}\right)$ is Kähler and $L_{G, a}$ is a holomorphic vector bundle over $M_{G, a}$. We can now state our main result as follows, where we still use the superscript $G$ to denote the $G$-invariant part.

Theorem 0.1. In the case where $(M, \omega, J)$ is Kähler, if $\mu^{-1}(0)$ is nonempty, then there exists an open neighborhood $\mathbb{O}$ of $0 \in \mathrm{g}^{*}$ such that for any regular value $a \in \mathbb{O}$ of $\mu$ with $\mu^{-1}(a)$ nonempty, the following Morse type inequality for Dolbeault cohomologies holds for any integer $p$,

$$
\begin{aligned}
& \operatorname{dim} H^{0, p}(M, L)^{G}-\operatorname{dim} H^{0, p-1}(M, L)^{G}+\cdots+(-1)^{p} \operatorname{dim} H^{0,0}(M, L)^{G} \\
& \leq \operatorname{dim} H^{0, p}\left(M_{G, a}, L_{G, a}\right)-\operatorname{dim} H^{0, p-1}\left(M_{G, a}, L_{G, a}\right) \\
& +\cdots+(-1)^{p} \operatorname{dim} H^{0,0}\left(M_{G, a}, L_{G, a}\right) .
\end{aligned}
$$

Theorem 0.1 still holds, for orbifolds, when $G$ does not act on $\mu^{-1}(a)$ freely. It reduces to the results proved in [TZ1,2] when $a=0$ is a regular value of $\mu$. Also, if we drop the assumption in Theorem 0.1 that $(M, \omega, J)$ is Kähler, then we get

\footnotetext{
${ }^{1}$ However, if $a \neq 0$, then $L_{G, a}$ is in general no longer a prequantum line bundle over $\left(M_{G, a}, \omega_{G, a}\right)$.
} 
as in [TZ3] the following singular quantization formula of Meinrenken-Sjamaar [MS] which extends (0.3),

$$
\operatorname{dim} Q(M, L)^{G}=\operatorname{dim} Q\left(M_{G, a}, L_{G, a}\right) .
$$

We organize the rest of this note as follows. In Section 1, we prove an easy result which will play an essential role in the proof of Theorem 0.1. In Section 2, we prove Theorem 0.1.

\section{An inequality for $a \in \mathrm{g}^{*}$ sufficiently close to zero}

In this section, we prove an easy estimate which is essential for the proof in the next section. In order to explain the basic ideas clearer, we will first treat the case where $G$ is abelian in a) and then prove the remaining $G$ nonabelian case in b).

a) The abelian case. In this subsection, we assume that $G$ is a torus.

For any $a \in \mathbf{g}^{*}$, set

$$
\begin{array}{r}
\mu_{a}=\mu-a, \\
\mathcal{H}_{a}=|\mu-a|^{2}=\left|\mu_{a}\right|^{2} .
\end{array}
$$

Proposition 1.1. There is an open neighborhood $\mathbb{O}$ of $0 \in \mathbf{g}^{*}$ such that for any $a \in \mathbb{O}$, the following inequality holds at each critical point $x$ of $\mathcal{H}_{a}$,

$$
\langle\mu(x)-a, \mu(x)\rangle \geq 0 .
$$

Proof. We will use Kirwan's geometric characterization [K1] of the critical point set of the norm square of moment maps to prove (1.2).

As in $[\mathrm{K} 1,3.4]$, denote by $\mathbb{A} \subset \mathbf{g}^{*}$ the finite set of weights associated to $\mu$, which consists of the values of $\mu$ taking on the fixed point set of $G$ on $M$. We also denote by $\mathbb{Y}$ the set of convex hulls in $\mathbf{g}^{*}$ generated by nonempty subsets of $\mathbb{A}$. Then $\mathbb{Y}$ consists naturally of two parts: the part $\mathbb{Y}_{I}$ consists of all those convex hulls not containing 0 , and the rest part denoted by $\mathbb{Y}_{I I}$.

Now let $U_{\delta}$ be an open ball in $\mathbf{g}^{*}$ with center 0 and radius $\delta>0$ such that the closure $\bar{U}_{\delta}$ does not intersect with any convex hull in $\mathbb{Y}_{I}$. The existence of $U_{\delta}$ is clear. Set $\mathbb{O}=U_{\delta / 2}$

Let $a \in \mathbb{O}$. Let $\mathbb{A}_{a}=\{A-a: A \in \mathbb{A}\}$ be the finite set of weights associated to $\mu_{a}$, and $\mathbb{Y}_{a}=\{Y-a: Y \in \mathbb{Y}\}$ the associated set of convex hulls. Then $\mathbb{Y}_{a}$ consists of two parts accordingly: $\mathbb{Y}_{I, a}=\left\{Y-a: Y \in \mathbb{Y}_{I}\right\}$ and $\mathbb{Y}_{I I, a}=\{Y-a$ : $\left.Y \in \mathbb{Y}_{I I}\right\}$. One verifies easily that the closure $\overline{\mathbb{O}}$ does not intersect with any convex hull in $\mathbb{Y}_{I, a}$.

Let $B_{a}$ be the open ball $B_{a}=\left\{y \in \mathbf{g}^{*}:\left|y+\frac{a}{2}\right|<\left|\frac{a}{2}\right|\right\}$. Clearly, $B_{a} \subset \mathbb{O}$. Thus $B_{a}$ does not intersect with any convex hull in $\mathbb{Y}_{I, a}$.

Now take $Y \in \mathbb{Y}_{I I, a}$. Let $y \in Y$ be the (unique) point on $Y$ which is closest to 0 . We claim that $y \in \mathbf{g}^{*} \backslash B_{a}$. 
To prove this claim, we suppose on the contrary that $y \in B_{a}$. Let $x \in \partial B_{a}$, with $x \neq-a$, lie in the straight line generated by $y$ and $-a \in \partial B_{a} \cap Y$, then it is easy to see that $x \in Y$. For if $x \notin Y$, then $y$ should lie in a face of $Y$ which does not contain $-a$. This would imply that $y$ lies in a convex hull in $\mathbb{Y}_{I, a}$, a contradiction. But with such an $x \in \partial B_{a} \cap Y$, one encounters another contradiction $d(0, x)<d(0, y)$. Thus we should have $y \notin B_{a}$.

By this and by the result of Kirwan [K1,3.12], one finds that if $y$ is a critical point of $\mathcal{H}_{a}$, then

$$
0 \leq\left|\mu_{a}(y)+\frac{a}{2}\right|^{2}-\left|\frac{a}{2}\right|^{2}=\langle\mu(y)-a, \mu(y)\rangle,
$$

which is exactly (1.2).

b) The nonabelian case. In this subsection, we no longer assume that $G$ is abelian.

Let $T$ be a maximal torus of $G$, with Lie algebra $\mathbf{t}$. Then $\mu_{T}=P_{T} \mu$, where $P_{T}$ is the orthogonal projection from $\mathbf{g}^{*}$ to $\mathbf{t}^{*}$, is the moment map of the induced $T$ action on $(M, \omega)$ (cf. [K1, 3.3]). Let $\mathbb{O}_{T} \subset \mathbf{t}^{*}$ be the open set defined in a) for $\mu_{T}$. Then $\mathbb{O}=\operatorname{Ad} G\left(\mathbb{O}_{T}\right) \subset \mathbf{g}^{*}$ is an open neighborhood of $0 \in \mathbf{g}^{*}$.

Take $a \in \mathbb{O}$. Recall that the coadjoint orbit $\mathcal{O}_{a}$ admits a canonical symplectic (actually Kähler) form $\omega_{a}$ and the (holomorphical) Ad $G$ action on $\mathcal{O}_{a}$ is Hamiltonian with the moment map given by the canonical embedding $i_{a}: \mathcal{O}_{a} \hookrightarrow \mathbf{g}^{*}$ (cf. [McS, Chap. 5]).

We now form the symplectic product $\left(M \times \mathcal{O}_{a}, \omega \times\left(-\omega_{a}\right)\right)$. Then the induced action of $G$ on $M \times \mathcal{O}_{a}$ is Hamiltonian with the moment map $\mu_{a}: M \times \mathcal{O}_{a} \rightarrow \mathbf{g}^{*}$ given by

$$
\mu_{a}(x, b)=\mu(x)-b
$$

Set

$$
\mathcal{H}_{a}=\left|\mu_{a}\right|^{2} .
$$

We can now state the main result of this section as follows.

Proposition 1.2. If $(x, b) \in M \times \mathcal{O}_{a}$ is a critical point of $\mathcal{H}_{a}$, then one has the following inequality for the inner product on $\mathbf{g}^{*}$,

$$
\langle\mu(x)-b, \mu(x)\rangle \geq 0 .
$$

Proof. Without loss of generality, we can assume that $a \in \mathbb{O}_{T}$. By a result of Kirwan [K2, pp. 551], we know that for any critical point $(x, b) \in M \times \mathcal{O}_{a}$ of $\mathcal{H}_{a}$, one can find $y \in \mathbf{t}^{*}$ such that $(y, a)$ is a critical point of $\mathcal{H}_{a}$ in the $G$-orbit of $(x, b)$.

Now since $a \in \mathbb{O}_{T}$, one finds from Proposition 1.1 and from $[\mathrm{K} 1,3.3]$ that

$$
\langle\mu(y)-a, \mu(y)\rangle \geq 0 .
$$


(1.6) then follows from the Ad $G$-invariance of the inner product on $\mathbf{g}^{*}$.

Remark 1.3. Propositions 1.1 and 1.2, while trivial, were first proved in a former preprint [TZ3], which will not be further submitted for publication.

\section{A proof of Theorem 0.1}

This section consists of two parts. In a), we state a system of holomorphic Morse inequalities valid for general vector bundles, which follows immediately from the arguments in [TZ2]. In b), we combine the result in a) and Proposition 1.2 to complete the proof of Theorem 0.1 .

In this section, we assume $(M, \omega, J)$ is a closed Kähler manifold and that the Hamiltonian $G$ action acts holomorphically on it.

a) Holomorphic Morse inequalities for general coefficients. In this subsection, we assume that $0 \in \mathbf{g}^{*}$ is a regular value of $\mu$ and, for simplicity, that $G$ acts on $\mu^{-1}(0)$ freely. Then one can construct the canonical Marsden-Weinstein reduction $\left(M_{G}=\mu^{-1}(0) / G, \omega_{G}\right)$ which is still Kähler (cf. [GS]).

Let now $E$ be a holomorphic Hermitian vector bundle, with the holomorphic connection denoted by $\nabla^{E}$, over $M$. We make the assumption that the $G$ action on $M$ lifts to a holomorphic $G$ action on $E$ which preserves the Hermitian metric and also the holomorphic Hermitian connection $\nabla^{E}$. Then $E$ descends canonically to a holomorphic Hermitian vector bundle $E_{G}$ over $M_{G}$.

Let $h_{i}, 1 \leq i \leq \operatorname{dim} G$, be an orthonormal base of $\mathbf{g}^{*}$. Let $V_{i}, 1 \leq i \leq \operatorname{dim} G$, be the dual base of $h_{i}, 1 \leq i \leq \operatorname{dim} G$. Then the moment map $\mu$ can be written as

$$
\mu=\sum_{i=1}^{\operatorname{dim} G} \mu_{i} h_{i}
$$

with each $\mu_{i}$ a real function on $M$. For any $V \in \mathbf{g}$, we use the same notation to denote the vector field it generates on $M$.

Now take $V \in \mathbf{g}$. Set

$$
r_{V}^{E}=L_{V}^{E}-\nabla_{V}^{E}
$$

where $L_{V}^{E}$ denotes the infinitesimal action of $V$ on $E$.

By using the arguments in [TZ2, Sect. 4d)], one gets immediately the following holomorphic refinement of [TZ2, Theorem 4.2].

Theorem 2.1. If $\mu^{-1}(0)$ is nonempty and if at each critical point $x \in M \backslash \mu^{-1}(0)$ of $\mathcal{H}$,

$$
\sqrt{-1} \sum_{i=1}^{\operatorname{dim} G} \mu_{i}(x) r_{V_{i}}^{E}(x) \geq 0
$$


then the following Morse type inequality for Dolbeault cohomologies holds for any integer $p$,

$$
\begin{aligned}
\operatorname{dim} & H^{0, p}(M, E)^{G}-\operatorname{dim} H^{0, p-1}(M, E)^{G} \\
& +\cdots+(-1)^{p} \operatorname{dim} H^{0,0}(M, E)^{G} \\
\leq & \operatorname{dim} H^{0, p}\left(M_{G}, E_{G}\right)-\operatorname{dim} H^{0, p-1}\left(M_{G}, E_{G}\right) \\
& +\cdots+(-1)^{p} \operatorname{dim} H^{0,0}\left(M_{G}, E_{G}\right) .
\end{aligned}
$$

b) A proof of Theorem 0.1. In this subsection, we no longer assume that $0 \in \mathbf{g}^{*}$ is a regular value of $\mu$.

Take a regular value $a \in \mathbb{O}$ of $\mu$ as defined in Section 1b). For simplicity, we assume $G$ acts on $\mu^{-1}\left(\mathcal{O}_{a}\right)$ freely. Then $0 \in \mathrm{g}^{*}$ is a regular value of $\mu_{a}$. Furthermore, one has the standard identification of the symplectic quotients

$$
\mu_{a}^{-1}(0) / G \equiv \mu^{-1}\left(\mathcal{O}_{a}\right) / G=M_{G, a},
$$

which carries a canonically induced symplectic form $\omega_{G, a}$ (cf. [McS, Chap. 5]). Furthermore, since $G$ acts on $M, \mathcal{O}_{a}$ and thus $M \times \mathcal{O}_{a}$ holomorphically, $\left(M_{G, a}, \omega_{G, a}\right)$ is again Kähler.

Let $\pi$ denote the projection from $M \times \mathcal{O}_{a}$ to its first factor $M$. Let $\mathcal{L}=\pi^{*} L$ be the pull-back holomorphic Hermitian line bundle over $M \times \mathcal{O}_{a}$ with the pull-back holomorphic Hermitian connection $\nabla^{\mathcal{L}}$ on $\mathcal{L}$ verifying that

$$
\frac{\sqrt{-1}}{2 \pi}\left(\nabla^{\mathcal{L}}\right)^{2}=\pi^{*} \omega
$$

Furthermore, the $G$ action on $L$ lifts canonically to a holomorphic action on $\mathcal{L}$. In particular, for any $V \in \mathbf{g}$, its induced infinitesimal action on $\mathcal{L}$ is, via the Kostant formula [Ko] (cf. [TZ2, (1.13)]) for the g-action on $L$, given by

$$
L_{V}^{\mathcal{L}}=\nabla_{V}^{\mathcal{L}}-2 \pi \sqrt{-1}\langle\mu \pi, V\rangle,
$$

from which one has, in using the notation in (2.2), that

$$
r_{V_{i}}^{\mathcal{L}}=-2 \pi \sqrt{-1} \mu_{i}(x)
$$

at any point $(x, b) \in M \times \mathcal{O}_{a}$. By $(2.8)$ and Proposition 1.2, one verifies that at any critical point $(x, b) \in M \times \mathcal{O}_{a}$ of $\mathcal{H}_{a}$,

$$
\sum_{i=1}^{\operatorname{dim} G} \sqrt{-1} \mu_{a, i}(x, b) r_{V_{i}}^{\mathcal{L}}(x, b)=2 \pi\langle\mu(x)-b, \mu(x)\rangle \geq 0 .
$$

One the other hand, one verifies directly that the induced line bundle $\mathcal{L}_{G}$ over $\mu_{a}^{-1}(0) / G$ is exactly the line bundle $L_{G, a}$ over $M_{G, a}=\mu^{-1}\left(\mathcal{O}_{a}\right) / G$. 
One can then apply Theorem 2.1 to $M \times \mathcal{O}_{a}, \mu_{a}$ and $\mathcal{L}$ to get

$$
\begin{aligned}
\operatorname{dim} H^{0, p}\left(M \times \mathcal{O}_{a}, \mathcal{L}\right)^{G}-\operatorname{dim} H^{0, p-1}\left(M \times \mathcal{O}_{a}, \mathcal{L}\right)^{G} \\
\quad+\cdots+(-1)^{p} \operatorname{dim} H^{0,0}\left(M \times \mathcal{O}_{a}, \mathcal{L}\right)^{G} \\
\leq \operatorname{dim} H^{0, p}\left(M_{G, a}, L_{G, a}\right)-\operatorname{dim} H^{0, p-1}\left(M_{G, a}, L_{G, a}\right) \\
\quad+\cdots+(-1)^{p} \operatorname{dim} H^{0,0}\left(M_{G, a}, L_{G, a}\right)
\end{aligned}
$$

for any integer $p$. Furthermore, by the definition of $\mathcal{L}$, one verifies that

$$
\begin{aligned}
\operatorname{dim} H^{0, p}\left(M \times \mathcal{O}_{a}, \mathcal{L}\right)^{G} & =\sum_{i+j=p} \operatorname{dim}\left(H^{0, i}(M, L) \otimes H^{0, j}\left(\mathcal{O}_{a}, \mathbb{C}\right)\right)^{G} \\
& =\operatorname{dim} H^{0, p}(M, L)^{G},
\end{aligned}
$$

which follows easily from the standard facts that

$$
\begin{aligned}
& \operatorname{dim} H^{0,0}\left(\mathcal{O}_{a}, \mathbb{C}\right)=\operatorname{dim} H^{0,0}\left(\mathcal{O}_{a}, \mathbb{C}\right)^{G}=1, \\
& \operatorname{dim} H^{0, j}\left(\mathcal{O}_{a}, \mathbb{C}\right)=0 \text { for } j \geq 1 .
\end{aligned}
$$

(0.4) follows from (2.10)-(2.12). The proof of Theorem 0.1 is completed.

\section{Acknowledgement}

We thank the referee for very helpful suggestions.

\section{References}

[AS] M. F. Atiyah and I. M. Singer, The index of ellitptic operators, III, Ann. of Math. 87 (1968), 546-604.

[BL] J.-M. Bismut and G. Lebeau, Complex immersions and Quillen metrics, Publ. Math. IHES. 74 (1991), 1-297.

[DGMW] H. Duistermaart, V. Guillemin, E. Meinrenken, and S. Wu, Symplectic reduction and Riemann-Roch for circle actions, Math. Res. Lett. 2 (1995), 259-266.

[G] V. Guillemin, Reduced phase space and Riemann-Roch, Lie Groups and Geometry, R. Brylinski et al. ed., Birkhaeuser (Progress in Math., 123), 1995, pp. 305-334.

[GS] V. Guillemin and S. Sternberg, Geometric quantization and multiplicities of group representations, Invent. Math. 67 (1982), 515-538.

[JK] L. C. Jeffrey and F. C. Kirwan, Localization and quantization conjecture, Topology 36 (1997), 647-693.

[K1] F. C. Kirwan, Cohomology of quotients in symplectic and algebraic geometry, Princeton Univ. Press, Princeton, 1984.

[K2] Convexity properties of the moment mapping, III, Invent. Math. 77 (1984), 547-552.

[Ko] B. Kostant, Quantization and unitary representations, Modern Analysis and Applications, Lecture Notes in Math. vol. 170, Springer-Verlag, (1970), pp. 87-207.

[M1] E. Meinrenken, On Riemann-Roch formulas for multiplicities, J. Amer. Math. Soc. 9 (1996), 373-389.

[M2] _ Symplectic surgery and the Spinc-Dirac operator, Adv. in Math. (to appear).

[MS] E. Meinrenken and R. Sjamaar, Singular reduction and quantization, Preprint, 1996 (dg-ga 9707023). 
[McS] D. Mcduff and D. Salamon, Introduction to symplectic topology, Clarendon Press, Oxford, 1995.

[TZ1] Y. Tian and W. Zhang, Symplectic reduction and quantization, C. R. Acad. Sci. Paris, Série I 324 (1997), 433-438.

[TZ2] - An analytic proof of the geometric quantization conjecture of GuilleminSternberg, Invent. Math. 132 (1998), 229-259.

[TZ3] _ Quantization formula for singular reductions, Preprint, 1997 (dg-ga 9706016).

[V] M. Vergne, Multiplicity formula for geometric quantization, I, II, Duke Math. J. 82 (1996), 143-179, 181-194.

Courant Institute of Mathematical Sciences, New York, NY 10012, U. S. A.

E-mail address: ytian@cims.nyu.edu

Nankai Institute of Mathematics, Tianjin, 300071, P. R. China

E-mail address: weiping@sun.nankai.edu.cn 